\title{
Learning, plasticity, and atypical generalization in children with autism
}

\author{
Barbara A. Church • Courtney L. Rice - Alexander Dovgopoly • \\ Christopher J. Lopata • Marcus L. Thomeer • Andrew Nelson • \\ Eduardo Mercado III
}

Published online: 6 January 2015

(C) Psychonomic Society, Inc. 2014

\begin{abstract}
Individuals with autism spectrum disorder (ASD) show accelerated learning in some tasks, degraded learning in others, and distinct deficits when generalizing to novel situations. Recent simulations with connectionist models suggest that deficits in cortical plasticity mechanisms can account for atypical patterns of generalization shown by some children with ASD. We tested the surprising theoretical prediction, from past simulations, that the children with ASD who show atypical generalization in perceptual categorization tasks will benefit more from training with a single prototypical member of the category than from training with multiple examples, but children with ASD who generalize normally will be comparatively harmed. The experimental results confirmed this prediction, suggesting that plasticity deficits may well underlie the difficulties that some children with ASD have generalizing skills, and these deficits are not specific to the acquisition of social skills, but rather reflect a more general perceptual learning deficit that may impact many abilities.
\end{abstract}

Keywords Autism · Category learning $\cdot$ Perceptual learning $\cdot$ Neural network modeling

Electronic supplementary material The online version of this article (doi:10.3758/s13423-014-0797-9) contains supplementary material, which is available to authorized users.

B. A. Church $(\bowtie) \cdot$ C. L. Rice $\cdot$ A. Dovgopoly $\cdot$ E. Mercado III Department of Psychology, Park Hall, University at Buffalo, The State University of New York, Buffalo, New York 14260, USA e-mail: bchurch@buffalo.edu

C. J. Lopata $\cdot$ M. L. Thomeer Institute for Autism Research, Canisius College, 2001 Main Street, Buffalo, NY 14208, USA

\section{A. Nelson}

Department of Counseling, School and Educational Psychology, University at Buffalo, The State University of New York, Buffalo, NY 14260, USA
Individuals with autism spectrum disorders (ASD) show enhanced perceptual discrimination abilities in multiple modalities, but often are impaired in perceptual processes that require complex configural or dynamic information. These individuals also may show accelerated learning and enhanced perceptual acuity in some tasks (e.g., O'Riordan \& Plaisted, 2001; Sears, Finn, \& Steinmetz, 1994) but distinct deficits in perceptual learning and the ability to generalize learning (Klinger \& Dawson, 2001; Plaisted, O'Riordan, \& BaronCohen, 1998). A number of researchers have proposed that perceptual deficits may reflect abnormalities in cortical structure and function (e.g., Casanova, Buxhoeveden, Switala, \& Roy, 2002, abnormal minicolumnar structure; Just, Cherkassky, Keller, \& Minshew, 2004, cortical underconnectivity; Kana, Libero, \& Moore, 2011, disrupted connectivity; Markram \& Markram, 2010, hyperplasticity; Rubenstein \& Merzenich, 2003, abnormal excitation/ inhibition ratios).

Individuals with ASD often have difficulty transferring learning to novel contexts (e.g., Mottron \& Burack, 2006, for review) and show atypical visual perceptual learning and generalization (Plaisted et al., 1998). In the area of perceptual categorization and generalization findings have been conflicting, with some researchers reporting deficits in the use of prototypes in individuals with ASD (Church et al., 2010, atypical generalization in random-dot-pattern shapes, children; Gastgeb, Rump, Best, Minshew, \& Strauss, 2009, abnormal learning of face prototypes, children and adults; Klinger \& Dawson, 2001, atypical learning of prototypes, children), and others reporting normal prototype effects (Bott, Brock, Brockdorff, Boucher, \& Lamberts, 2006, sensitivity to exemplars in categorization, adults; Molesworth, Bowler, \& Hampton, 2005, recognition of prototype, children; 2008, category learning, children; Froehlich et al., 2012; Gastgeb, Dundas, Minshew, \& Strauss, 2012; Schipul, 2012; Vladusich, Olu-Lafe, Kim, Tager-Flusberg, \& Grossberg, 
2010, random-dot-pattern classification, teenagers and adults; Soulières, Mottron, Giguère, \& Larochelle, 2011, rule and exemplar learning, ages unspecified). However, even when apparently normal categorization is observed, individuals with high-functioning ASD (HFASD) showed impairments in learning or generalization (Bott et al., 2006; Gastgeb et al., 2012; Froehlich et al., 2012; Soulières et al., 2011; Vladusich et al., 2010), differences in brain activity (Schipul, 2012), or subgroups who showed abnormal generalization (Molesworth et al., 2008), suggesting that the neural mechanisms or processes involved may differ. This contrasts with relatively consistent findings of spared simple explicit rule formation and implicit associative/statistical learning in individuals with ASD (e.g., Brown, Aczel, Jimenez, Kaufman, \& Grant, 2010, contextual cueing, serial reaction time, artificial grammar learning, and probabilistic classification; Klinger and Dawson, 2001, single-dimensional rules).

Given this complexity, computational models may help to clarify the deficits mediating atypical perceptual category learning in ASD. Recent simulations using a neural network $(\mathrm{NN})$ model of visual processing (Henderson \& McClelland, 2011) to investigate the possible effects of neural abnormalities on visual category learning suggest that atypical generalization may reflect disrupted cortical reorganization resulting in slower perceptual learning in some children with HFASD (Dovgopoly \& Mercado, 2013). These simulations replicated atypical generalization patterns (Church et al., 2010) by taking into account two subgroups of participants with HFASD: typical learners (A Type 1) who show no differences in performance from typically developing children and atypical learners (A Type 2). Generalization similar to A Type 2 children was shown by NNs with reduced plasticity (the ability to change connections with experience) or disrupted synaptic homeostasis (the processes regulating the amount of synaptic change). In contrast to prior computational models of abnormal processing in ASD, this model makes quantitative predictions of generalization patterns for specific stimuli and training regimens.

Simulations with this NN model led to the novel and counterintuitive prediction that the subgroup of individuals with HFASD who are atypical learners in a standard task will perform better when trained with highly simplified input sets consisting of a single prototypical exemplar rather than multiple variable examples of the category (Dovgopoly \& Mercado, 2013). This occurs because repeated experiences with an "ideal" stimulus, combined with degraded plasticity, leads to an internal representation that readily generalizes to distortions of the prototype, but not random patterns. In contrast, typically developing (TD) children and adults generally show reduced generalization after such training and better learning with variable training examples (e.g., Hayes \& Taplin, 1993, school age children; Homa, Sterling, \& Trepel, 1981, adults; Perry, Samuelson, Malloy, \& Schiffer, 2010, toddlers; see also supplementary materials $\{\mathrm{SM}\}$ and Figure S1). Importantly, although simulations of either disrupted plasticity or abnormal homeostasis predict that training with prototypes can be beneficial, they make different predictions about the specific generalization that will occur after such training (Fig. 1). Thus, tests of the effects of prototype training in children with HFASD who show atypical learning can be used to assess the predictive utility of the $\mathrm{NN}$ model, and provide a way to evaluate whether a simulated deficit in either neural plasticity or synaptic homeostasis better predicts the specific generalization patterns observed. The present study was designed to test these predictions.
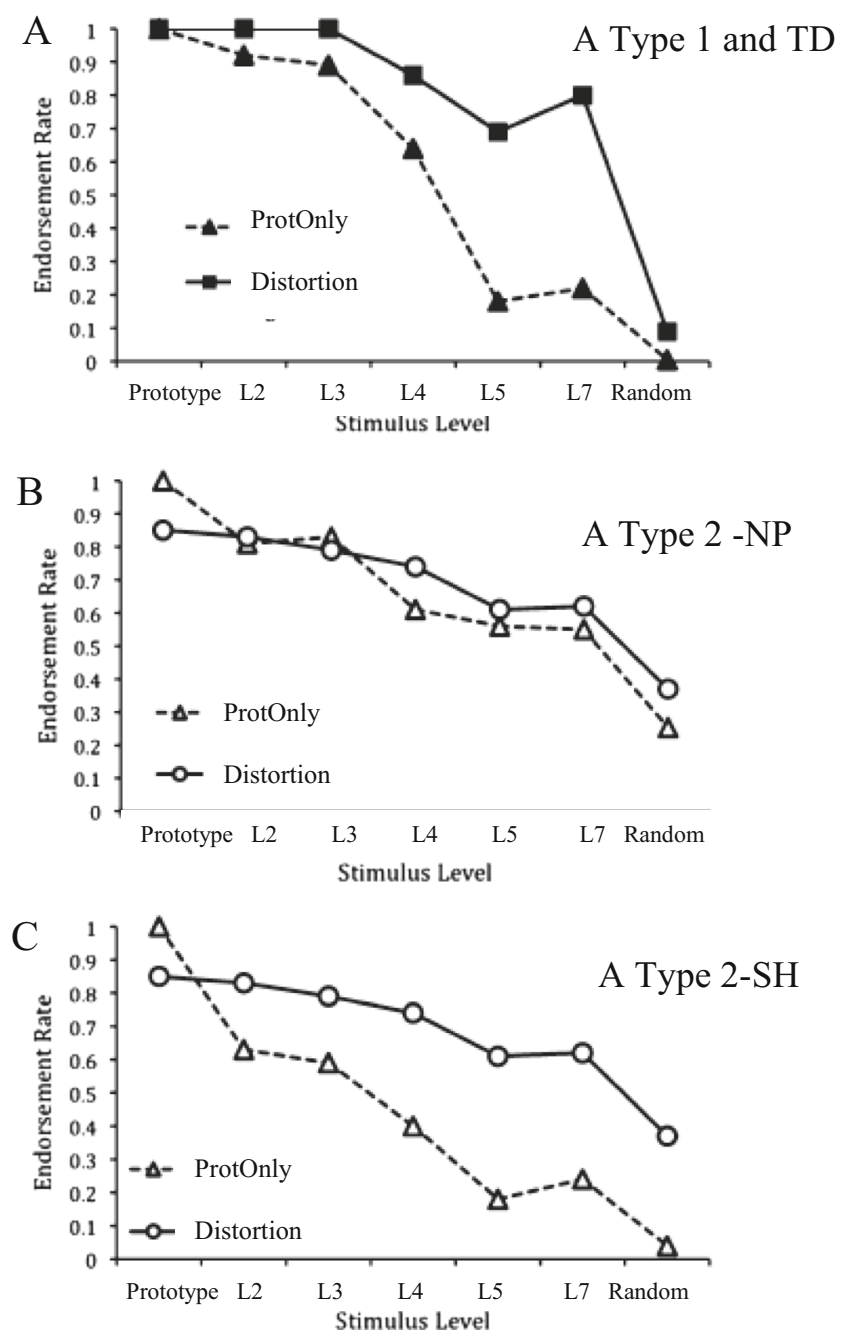

Fig. 1 a Proportion of category endorsements across stimulus type after Distortion training for neural networks (NNs) configured to perform like TD and A Type 1 children (solid line) and proportions predicted by these same neural networks after Prototype-Only training. b Proportion of category endorsements after Distortion training for NNs configured to generalize like A Type 2 children (solid line) by simulating disrupted neural plasticity and proportions predicted by these same NNs after Prototype-Only training. c Proportion of endorsements after Distortion training for NNs configured to generalize like A Type 2 children by simulating disrupted synaptic homeostasis and proportions predicted by these same neural networks after Prototype-Only training 
Children with HFASD classified complex shapes as members of a category or not after training involving either multiple examples or a single prototype. Within-participant variations in generalization patterns with each type of training were compared with patterns predicted by the NN model.

\section{Methods}

Participants Forty-three children with HFASD, ages 7 to 12 years, participated. Two participants were omitted for having more than four missing values, one for response bias (more than $80 \%$ ), one for patterned responding (left-right alternation), and three were randomly dropped to equate counterbalancing conditions. Thirty-six children were included in the final analyses. Children were recruited from a summer psychosocial intervention program for children with HFASD. The children met diagnosis, intelligence, and language criteria to be in the program (see SM).

Stimuli Our modified versions of the random-dot-pattern classification task used Posner shapes. Posner shapes are created by taking a random 9-dot pattern and connecting the dots with lines in the order the dots are chosen. The resulting shapes possess all the similarity relationships of random-dot-patterns, but are more visually engaging. Shapes within a category were constructed by creating a random prototype, and then generating different levels of distortion from that prototype (See SM and Table S1 for construction details). Nonmembers were new random shapes dissimilar from the prototype. Colors varied randomly, and participants were informed that color was irrelevant (Figure S2).

Two randomly generated prototype shapes, 40 distorted versions of each of these prototypes that varied in distortion level (L-2, L-3, L-4, L-5, \& L-7, where higher numbers indicate greater distortion), and 90 (45 used with each stimulus set) randomly generated non-category members were created.

Materials IBM-compatible laptop computers were used for testing. Stimuli were presented, responses collected, and feedback given using DMDX experimental software (Forster \& Forster, 2003).

Procedure and design We used a $(2 \times 7)$ mixed factorial design with category endorsement as the dependent measure. Training condition (Distortion or Prototype-Only), and stimulus type (prototype, L2, L3, L4, L5, L7, and random) were the within participant independent variables with 2 and 7 levels, respectively.

Each trial consisted of one shape presented in the middle of a computer screen against a black background and two icons presented on the top left and right. The icon presented on the left was a red circle with a line through it. The icon presented on the right was either a picture of a castle (with stimulus set A) or a cave (with stimulus set B). Participants responded by pushing the button marked $\mathrm{N}$ on the left (category exclusion) or $\mathrm{Y}$ on the right (category endorsement).

The children were tested individually in a quiet room at their summer treatment program. Four children stationed at different desks with dividers between them were tested at a time. The experiment lasted about half an hour. Every child participated in two conditions (one with Prototype-Only training, and one with variable Distortion training). Both conditions had training and testing phases. The training varied depending on the condition (described below). The test was the same across conditions. Participants learned a new category for each condition (Category A and Category B).

Participants received verbal instructions and written reminders about the goal of the task. Each condition lasted approximately 15 minutes. Children received a cover story that the shapes were ghosts and their job each time was to hunt down the ghosts of a particular type. They were directly told that for each hunt, the ghosts were different kinds (e.g., castle ghosts and cave ghosts), and any type of ghost could be any color; so they should not make any decisions based on color. The order of the conditions and particular stimulus set used for each condition were fully counterbalanced across children. The same children also participated in a different experiment using other categorization tasks with different stimuli on the same day. In order to assure that no practice or fatigue effects from the other experiment could confound the results, equal numbers of children in the Prototype-Only training first and Distortion training first conditions participated in this experiment first or second.

During training, participants had to decide whether each shape was a member of the category. They viewed and responded to 30 shapes: half belonged to the category; half did not. The Distortion training involved training with level 3, 5 , and 7 distortions of the prototype (five shapes from each distortion level), as well as 15 randomly created nonmember shapes. In the Prototype-Only training condition, a prototype shape was presented 15 times along with 15 nonmember shapes. After producing a correct response, children were shown a dancing monkey. Following an incorrect response, they saw the shape move to the correct answer.

After training, participants were given a generalization test. They were asked to classify, without feedback, 60 shapes: five repetitions of the prototype, five each of new $2,3,4,5$, and 7 level distortions, and 30 new random shapes.

\section{Results}

The children with HFASD were classified into two groups based on the percentage of random shapes they endorsed after 
the distortion training $(\leq 30 \%=$ A Type $1 ;>30 \%=$ A Type 2$)$. Previous research showed that this percentage of endorsements of random shapes predicts the distinctive A Type 1 and A Type 2 generalization patterns seen in children with HFASD (Dovgopoly \& Mercado, 2013). Also, the TD children in our previous research (Church et al., 2010) meet this A Type 1 criterion. The endorsement proportions were analyzed using standard statistics and prototype modeling to examine the degree of learning and generalization.

Fifteen children were classified as A Type 1 and 21 children were classified as A Type 2 (see Table 1 for demographics). As previously shown (Dovgopoly \& Mercado, 2013), A Type 1 children showed generalization patterns similar to those previously found for TD children after training with distorted prototypes (Church et al., 2010). However, they showed relatively poorer generalization after training with the PrototypeOnly task (Fig. 2a). This training-dependent difference in generalization is also seen in undergraduates with these exact stimuli and tasks (see SM). In contrast, A Type 2 children showed poor generalization after Distortion training, and, in comparison, improved generalization after Prototype-Only

Table 1 Demographic characteristics for subgroups of children with HFASD $^{\mathrm{a}}$

\begin{tabular}{lll}
\hline Characteristic & A Type I $(n=15)$ & A Type II $(n=21)$ \\
\hline & Mean $(S D)$ & Mean $(S D)$ \\
Age (yr) & $10.27(1.58)$ & $9.43(1.72)$ \\
Parent education (yr) & $15.57(2.16)$ & $15.81(1.48)$ \\
WISC-IV Short Form IQ & $112.94(16.50)$ & $107.55(13.48)$ \\
$\quad$ Verbal IQ (VCI) & $110.04(16.90)$ & $105.23(13.91)$ \\
Performance IQ (PRI) & $113.03(16.66)$ & $107.84(14.92)$ \\
CASL & & \\
Expressive language & $106.20(16.48)$ & $103.33(14.91)$ \\
Receptive language & $108.20(13.49)$ & $105.74(13.47)$ \\
ADI-R & & \\
QARSI & $22.11(3.92)$ & $20.90(4.97)$ \\
QAC & $17.22(2.77)$ & $16.57(3.44)$ \\
RRSB & $5.55(3.21)$ & $6.71(2.70)$ \\
& $n(\%$ of total) & $n(\%$ of total $)$ \\
Gender & & $19(90.0)$ \\
Male & $13(87.0)$ & $2(10.0)$ \\
Female & $2(13.0)$ & $19(90.0)$ \\
Ethnicity & $14(93.0)$ & $2(10.0)$ \\
Caucasian & $1(7.0)$ & \\
African American & & \\
\hline
\end{tabular}

WISC-IV, Wechsler Intelligence Scale for Children-4th Edition; CASL, Comprehensive Assessment of Spoken Language; ADI-R, Autism Diagnostic Interview-Revised (QARSI, Qualitative Abnormalities in Reciprocal Social Interactions; QAC, Qualitative Abnormalities in Communication; RRSB, Restricted, Repetitive, and Stereotyped Patterns of Behavior)

${ }^{\mathrm{a}}$ There were no significant differences between A Type 1 and A Type 2 children on any measure, all $t$ 's $<1.5$

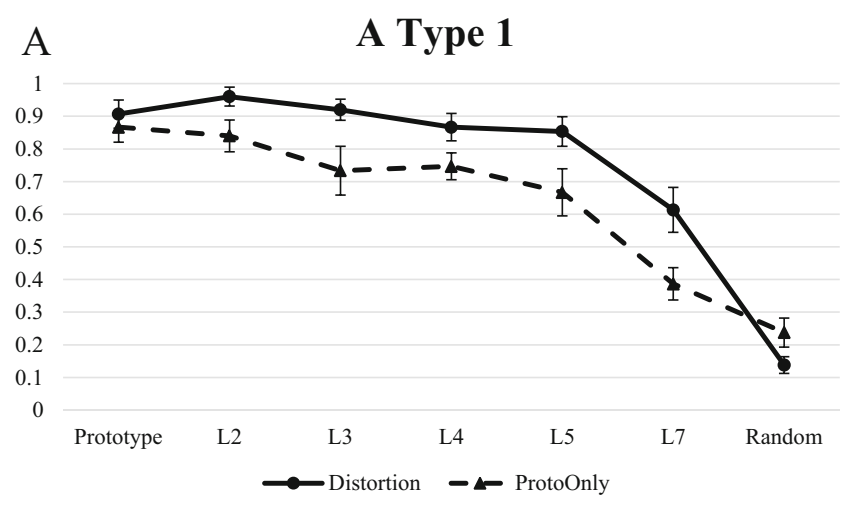

B

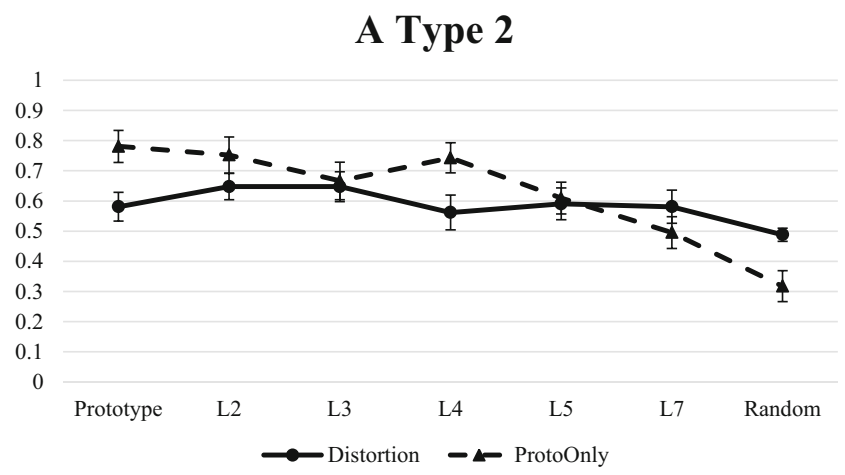

Fig. 2 a Proportion of category endorsements across stimulus type after Distortion and Prototype-Only training for A Type 1 children. b Proportion of category endorsements across stimulus type after Distortion and Prototype-Only training for A Type 2 children. All error bars depict standard errors of the means

training (Fig. 2b). The pattern of generalization after Prototype-Only training by A Type 2 children was more similar to generalization by NN models when plasticity was reduced than to generalization by NN models simulating disrupted synaptic homeostasis (Fig. 1).

A $2 \times(2 \times 7)$ GLM using the proportion of category endorsements as the dependent measure, A Type $(1,2)$ as the between participants factor and Training (Distortions, Prototype-Only) and Stimuli (prototype, L2, L3, L4, L5, L7, random) as the within participant factors found significant main effects of A Type, $F(1,34)=7.95, p=.008, \eta_{p}{ }^{2}=0.19$, and Stimuli, $F(6,204)=51.30, p<0.001, \eta_{p}{ }^{2}=0.60$. This reflected A Type 1 children's greater overall endorsement of ghosts and greater endorsement of lower-level distortions. The main effect of Training was not significant, $F(1,34)=2.37, p=$ $0.133, \eta_{p}{ }^{2}=0.07$, but the interactions between Training and $\mathrm{A}$ Type, $F(1,34)=9.88, p=0.003, \eta_{p}{ }^{2}=0.23$, Training and Stimuli, $F(6,204)=3.23, p=0.005, \eta_{p}{ }^{2}=0.06$, Stimuli and A Type, $F(6,204)=9.68, p<0.001, \eta_{p}{ }^{2}=0.22$, and Training, Stimuli, and A Type, $F(6,204)=4.69, p<0.001, \eta_{p}{ }^{2}=0.09$ were significant, showing that Training condition changed the pattern of endorsements, and that the changes varied depending on A Type. 
In order to better understand the interaction between Training, A Type group, and Stimuli, we completed two further analyses. In the first, we compared the overall percent of correct endorsements after each type of training for each group type. As predicted, A Type 2 children showed a significant increase in correct endorsements after Prototype-Only training compared with Distortion training, $t(20)=2.64, p=0.016, d=$ 0.23 . Also as predicted, A Type 1 children showed a significant decrease in correct categorization with Prototype-Only compared with Distortion training, $t(14)=4.31, p<0.001$, $d=0.44$.

In the second analysis, we further characterized the generalization patterns by modelling the data using a common prototype model (see SM). This model assumes participants make responses based on similarity comparisons between stimuli and the prototype. The model contains two free parameters, sensitivity and criterion, that are determined for each individual using a standard hill-climbing algorithm to find the values that allow the best fit. Sensitivity is meant to reflect how sensitive the participant is to perceptual differences between the stimuli and the prototype. Criterion is meant to reflect how similar the stimuli are to members of the category and members of other known categories. Fit is measured by calculating the sum of squared deviations (SSD) between the original and predicted data. The smaller the SSD, the better the fit.

We modelled the data of each participant individually and conducted planned comparisons of the Fit (SSD) and Sensitivity scores of A Type 1 and A Type 2 children in each training condition (Table 2). The comparisons found that, as expected given the inclusion criteria, A Type 2 participants had a significantly worse Fit to the prototype model and lower Sensitivity scores than A Type 1 children after Distortion training, $t(34)=3.24, p=0.003, d=1.09$, for Fit, and $t(34)=8.62$, $p<0.001, d=3.79$ for Sensitivity, but not after PrototypeOnly training $t(34)<1, d=0.31$ for Fit, and $t(34)=1.43, p=$ $0.118, d=0.55$ for Sensitivity. This reflected the improved Fit and Sensitivity after Prototype-Only training compared with Distortion training in the A Type 2 children, but like TD children and adults (e.g., Hayes \& Taplin, 1993; Homa et al., 1981; Perry et al., 2010; SM), A Type 1 children showed

Table 2 Means and standard deviations () of SSD fits and Sensitivity scores for A Type 1 and A Type 2 children in each training condition

\begin{tabular}{lll}
\hline Condition & SSD fit & Sensitivity score \\
\hline Distortion & & \\
A Type 1 & $0.09(0.09)$ & $2.02(0.66)$ \\
A Type 2 & $0.21(0.11)$ & $0.39(0.47)$ \\
Prototype-only & & \\
A Type 1 & $0.13(0.07)$ & $1.62(0.84)$ \\
A Type 2 & $0.17(0.15)$ & $1.13(0.93)$ \\
\hline
\end{tabular}

decreases in Fit and Sensitivity after Prototype-Only compared with Distortion training.

\section{Discussion}

In this study, we tested quantitative predictions derived from earlier neural network (NN) model simulations of visual category learning by children with HFASD. NNs predicted that children with HFASD who showed atypical generalization would benefit from training with a single prototypical example of the category, whereas children with HFASD who showed typical generalization would show the opposite effect. The simulations also predicted that the effects of PrototypeOnly training on generalization would differ depending on whether the source of the problem was reduced neural plasticity versus disruption of synaptic homeostasis. A large subset of children with HFASD in the current study showed deficits in generalization, consistent with earlier findings (Church et al., 2010). The children that showed deficits benefitted from Prototype-Only training compared to Distortion training, as predicted by the $\mathrm{NN}$ model, and they showed generalization patterns that were more consistent with dysfunctional neural plasticity than with disruptions in homeostasis.

Numerous qualitative and computational models have been developed to account for specific behavioral and neural abnormalities associated with ASD (for reviews, see Björne \& Balkenius, 2005; Dovgopoly \& Mercado, 2013). Most of these models make only general predictions about the behavioral or perceptual consequences of these abnormalities. The lack of theoretical frameworks providing precise predictions about when people with ASD will differ from TD individuals has led to vociferous debates about the nature of ASD as well as the underlying processes or neural abnormalities and their effect on learning (e.g., Casanova et al., 2002; Just, et al., 2004; Kana et al., 2011; Markram \& Markram, 2010; Rubenstein \& Merzenich, 2003; for review, Mottron \& Burack, 2006). Although most current behavioral strategies for ameliorating cognitive deficits associated with ASD assume that the individual's learning mechanisms are intact and comparable to those of TD individuals, numerous studies have demonstrated perceptual learning deficits, as well as instances of accelerated learning (see Dawson, Mottron, \& Gernsbacher, 2008, for review).

Several ASD researchers have noted that if the perceptual inputs individuals with ASD receive are abnormal, then the perceptual, cognitive, and social skills that they acquire over a lifetime of such aberrant experiences may differ substantially from those of TD individuals (e.g., Donnellan, Hill, \& Leary, 2013; Markram \& Markram, 2010). Similarly, if the neural plasticity mechanisms of perception are dysfunctional, this could also lead to dramatic differences in skill acquisition. Given that developmental trajectories reflect the learning- 
dependent construction of neural circuits (e.g., Quartz \& Sejnowski, 1997), it is clear that atypical perceptual learning mechanisms could lead to the atypical developmental patterns associated with ASD. Although researchers are becoming increasingly cognizant of this possibility, the potential effects of aberrant neural plasticity mechanisms on the perceptual, cognitive, and social abilities of children with ASD remain understudied.

As a first-step toward evaluating the potential role of neural abnormalities in atypical generalization by some individuals with ASD, Dovgopoly and Mercado (2013) conducted a series of simulations of visual cortical processing to assess the specific impacts of different neural deficits on the generalization of visual categories. Past computational research and theories regarding the role of abnormal cortical connectivity suggested that differences in the number of processing units engaged during learning might account for generalization deficits observed in children with ASD (e.g., Cohen, 1998). Instead, simulations by Dovgopoly and Mercado found that this factor could not account for atypical generalization, whereas deficits in neural plasticity or homeostasis (processes that modulate changes in connections over time) could be an explanation. The current results are consistent with the generalization patterns predicted by simulations of degraded neural plasticity mechanisms. Such specific predictions were only possible because this $\mathrm{NN}$ model was designed to capture specific elements of image processing and training regimens used in the experiments. Although the model focuses exclusively on visual cortical processing, and therefore does not make specific predictions about other perceptual or social events, an implicit prediction of the model is that any perceptual-motor, social, or cognitive process that is perceptual learningdependent could be affected by abnormal cortical plasticity. In particular, the model predicts that the more that ability depends on complex learning-induced changes in cortex, the more likely it will be that an individual with ASD differs from TD individuals in that ability.

Our findings suggest that the atypical perceptual category learning by some children with ASD can be explained by a deficit in neural plasticity, especially plasticity involved in modifications of perceptual processing. If a child with ASD is having problems learning to perceptually distinguish and conceptually organize sensory events, then early interventions that identify and redirect deviant perceptual and categorical learning trajectories may be invaluable in promoting better developmental outcomes. In particular, when children with ASD are generalizing atypically, simple prototypical learning examples may be beneficial.

Author note This research was supported by NSF grant \#SMA1041755 to the Temporal Dynamics of Learning Center, an NSF Science of Learning Center, Department of Education, Institute of Education Sciences Grant R324A080136, and by a grant from the Organization for
Autism Research. Conclusions are those of the authors and do not necessarily reflect the views of the funding agencies. The authors thank Matt Wisniewski, J. David Smith, Kanza Khalid, and the staff of the summerMAX treatment program for their help.

\section{References}

Björne, P., \& Balkenius, C. (2005). A model of attentional impairments in autism: First steps toward a computational theory. Cognitive Systems Research, 6, 193-204. doi:10.1016/j.cogsys.2004.11.003

Bott, L., Brock, J., Brockdorff, N., Boucher, J., \& Lamberts, K. (2006). Perceptual similarity in autism. Quarterly Journal of Experimental Psychology, 59, 1237-1254. doi:10.1080/02724980543000196

Brown, J., Aczel, B., Jimenez, L., Kaufman, S. B., \& Grant, K. P. (2010). Intact implicit learning in autism spectrum conditions. Quarterly Journal of Experimental Psychology, 63, 1789-1812. doi:10.1080/ 17470210903536910

Casanova, M. F., Buxhoeveden, D. P., Switala, A. E., \& Roy, E. (2002). Minicolumnar pathology in autism. Neurology, 58, 428-432. doi: 10.1212/wnl.58.3.428

Church, B. A., Krauss, M. S., Lopata, C., Toomey, J. A., Thomeer, M. L., Coutinho, M. V., \& Mercado, E., III. (2010). Atypical categorization in children with high-functioning autism spectrum disorder. Psychonomic Bulletin \& Review, 17, 862-868. doi:10.3758/PBR. 17.6.862

Cohen, I. L. (1998). Neural network analysis of learning in autism. In D. Stein \& J. Ludick (Eds.), Neural networks and psychopathology (pp. 274-315). Cambridge, U.K.: Cambridge University Press.

Dawson, M., Mottron, L., \& Gernsbacher, M. A. (2008). Learning in autism. In J. Byrne \& H. L. Roediger III (Eds.), Learning and memory: A comprehensive reference (Cognitive psychology, Vol. 2, pp. 759-772). Oxford, U.K.: Elsevier.

Donnellan, A. M., Hill, D. A., \& Leary, M. R. (2013). Rethinking autism: Implications of sensory and movement differences for understanding and support. Frontiers in Integrative Neuroscience, 6, 124. doi: 10.3389/fnint.2012.00124

Dovgopoly, A., \& Mercado, E., III. (2013). A connectionist model of category learning by individuals with high-functioning autism spectrum disorder. Cognitive, Affective, \& Behavioral Neuroscience, 13, 371-389. doi:10.3758/s13415-012-0148-0

Forster, K. L., \& Forster, J. C. (2003). DMDX: A windows display program with millisecond accuracy. Behavior Research Methods, Instruments, \& Computers, 35, 116-124. doi:10.3758/BF03195503

Froehlich, A. L., Anderson, J. S., Bigler, E. D., Miller, J. S., Lange, N. T., DuBray, M. B., \& Lainhart, J. E. (2012). Intact prototype formation but impaired generalization in autism. Research in Autism Spectrum Disorders, 6, 921-930. doi:10.1016/j.rasd.2011.12.006

Gastgeb, H. Z., Dundas, E. M., Minshew, N. J., \& Strauss, M. S. (2012). Category formation in autism: Can individuals with autism form categories and prototypes of dot patterns? Journal of Autism and Developmental Disorders, 42, 1694-1704. doi:10.1007/s10803011-1411-x

Gastgeb, H. Z., Rump, K. M., Best, C. A., Minshew, N. J., \& Strauss, M. S. (2009). Prototype formation in autism: Can individuals with autism abstract facial prototypes? Autism Research, 2, 279-284. doi: 10.1002/aur.93

Hayes, B. K., \& Taplin, J. E. (1993). Developmental differences in the use of prototype and exemplar-specific information. Journal of Experimental Child Psychology, 55, 329-352. doi:10.1006/jecp. 1993.1019

Henderson, C., \& McClelland, J. L. (2011). A PDP model of the simultaneous perception of multiple objects. Connection Science, 23, 161-172. doi:10.1080/09540091.2011.575931 
Homa, D., Sterling, S., \& Trepel, L. (1981). Limitations of exemplarbased generalization and the abstraction of categorical information. Journal of Experimental Psychology: Human Learning and Memory, 7, 418-439. doi:10.1037/0278-7393.7.6.418

Just, M. A., Cherkassky, V. L., Keller, T. A., \& Minshew, N. J. (2004). Cortical activation and synchronization during sentence comprehension in high-functioning autism: Evidence of underconnectivity. Brain, 127, 1811-1821. doi:10.1093/brain/awh199

Kana, R. K., Libero, L. E., \& Moore, M. S. (2011). Disrupted cortical connectivity theory as an explanatory model for autism spectrum disorders. Physics of Life Reviews, 8, 410-437. doi:10.1016/j. plrev.2011.10.001

Klinger, L. G., \& Dawson, G. (2001). Prototype formation in autism. Developmental Psychopathology, 13, 111-124. doi:10.1017/ S0954579401001080

Markram, K., \& Markram, H. (2010). The intense world theory - A unifying theory of the neurobiology of autism. Frontiers in Human Neuroscience, 4, 224. doi:10.3389/fnhum.2010.00224

Molesworth, C. J., Bowler, D. M., \& Hampton, J. A. (2005). The prototype effect in recognition memory: Intact in autism? Journal of Child Psychology and Psychiatry, 46, 661-672. doi:10.1111/j. 1469-7610.2004.00383.x

Molesworth, C. J., Bowler, D. M., \& Hampton, J. A. (2008). When prototypes are not best: Judgments made by children with autism. Journal of Autism and Developmental Disorders, 38, 1721-1730. doi:10.1007/s10803-008-0557-7

Mottron, L., \& Burack, J. (2006). Autism: A different perception. Journal of Autism and Developmental Disorders, 36, 1-3. doi:10.1007/ s10803-005-0048-Z

O'Riordan, M., \& Plaisted, K. (2001). Enhanced discrimination in autism. Quarterly Journal of Experimental Psychology. A,
Human Experimental Psychology, 54, 961-979. doi:10.1080/ 713756000

Perry, L. K., Samuelson, L. K., Malloy, L. M., \& Schiffer, R. N. (2010). Learn locally, think globally: Exemplar variability supports higherorder generalization and word learning. Psychological Science, 21, 1894-1902. doi:10.1177/0956797610389189

Plaisted, K., O'Riordan, M., \& Baron-Cohen, S. (1998). Enhanced discrimination of novel, highly similar stimuli by adults with autism during a perceptual learning task. Journal of Child Psychology and Psychiatry, 39, 765-775. doi:10.1017/ S0021963098002601

Quartz, S. R., \& Sejnowski, T. J. (1997). The neural basis of cognitive development: A constructivist manifesto. Behavioral and Brain Sciences, 20, 537-556. doi:10.1017/s0140525x97001581

Rubenstein, J. L., \& Merzenich, M. M. (2003). Model of autism: Increased ratio of excitation/inhibition in key neural systems. Genes, Brain and Behavior, 2, 255-267. doi:10.1046/j.1601-183X. 2003.00037.x

Schipul, S. E. (2012). Neural mechanisms of implicit and explicit learning in autism. Unpublished doctoral dissertation, Carnegie Mellon Universty, Pittsburgh, PA.

Sears, L. L., Finn, P. R., \& Steinmetz, J. E. (1994). Abnormal classical eye-blink conditioning in autism. Journal of Autism and Developmental Disorders, 24, 737-751. doi:10.1007/BF02172283

Soulières, I., Mottron, L., Giguère, G., \& Larochelle, S. (2011). Category induction in autism: Slower, perhaps different, but certainly possible. Quarterly Journal of Experimental Psychology, 64, 311-327. doi:10.1080/17470218.2010.492994

Vladusich, T., Olu-Lafe, O., Kim, D. S., Tager-Flusberg, H., \& Grossberg, S. (2010). Prototypical category learning in high-functioning autism. Autism Research, 3, 226-236. doi:10.1002/aur.148 